\title{
Après les retraites massives dans deux hôpitaux québécois : enjeux des rapports entre générations différentes de travailleurs
}

After the massive retirements in two quebecois hospitals: Toward a problematic of the relations between workers's cohorts

Después de jubilaciones masivas en dos hospitales quebequenses: desafíos de las relaciones entre generaciones diferentes de trabajadores

\section{Solange Lefebvre}

\section{(2) OpenEdition}

\section{Journals}

\section{Édition électronique}

URL : http://journals.openedition.org/pistes/3829

DOI : $10.4000 /$ pistes.3829

ISSN : 1481-9384

Éditeur

Les Amis de PISTES

Édition imprimée

Date de publication : 1 mai 2000

Référence électronique

Solange Lefebvre, "Après les retraites massives dans deux hôpitaux québécois : enjeux des rapports entre générations différentes de travailleurs », Perspectives interdisciplinaires sur le travail et la santé [En ligne], 2-1 | 2000, mis en ligne le 01 mai 2000, consulté le 19 avril 2019. URL : http:// journals.openedition.org/pistes/3829; DOI : 10.4000/pistes.3829

Ce document a été généré automatiquement le 19 avril 2019.

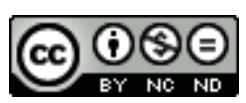

Pistes est mis à disposition selon les termes de la licence Creative Commons Attribution - Pas d'Utilisation Commerciale - Pas de Modification 4.0 International. 


\title{
Après les retraites massives dans deux hôpitaux québécois : enjeux des rapports entre générations différentes de travailleurs
}

\author{
After the massive retirements in two quebecois hospitals: Toward a problematic \\ of the relations between workers's cohorts \\ Después de jubilaciones masivas en dos hospitales quebequenses : desafíos de las \\ relaciones entre generaciones diferentes de trabajadores
}

Solange Lefebvre

\section{d'années :}

"Je le vois encore, ce jeune gestionnaire, se promener dans les corridors de l'établissement, et clamer à tous vents : Mon rêve, c'est qu'il n'y ait plus personne en haut de quarante ans dans cet hôpital! ».

\section{années 1960-1970 :}

Cette phrase n'est pas sans évoquer le fameux slogan de la révolution culturelle des

« Ne faites confiance à personne au-dessus de 30 ans »; ou encore celui-ci : «Plus personne ne transmet rien à personne » (Lefebvre, 1995).

C'est là indiquer dès le départ notre intérêt pour les rapports entre âge, expérience et formation.

Dans le domaine des recherches sur la santé et la sécurité au travail, les rapports entre les groupes d'âge de travailleurs demandent à être étudiés davantage. L'âge et la génération sont des axes d'analyse fondamentaux, au même titre que les sexes et les classes sociales par exemple. Pour fins de clarification, mentionnons que l'âge indique la position dans le cycle de vie ; il relève à la fois de conditions psychologiques, sociales et culturelles : le fait d'avoir vingt ans ou cinquante ans induit certaines caractéristiques spécifiques et définies selon ces trois dimensions. La génération réfère, pour la démographie, à une cohorte 
d'individus nés durant la même période; la sociologie examine ce que partagent ces individus pour constituer une mémoire historique collective (Attias-Donfut, 1991).

L'un des enjeux capitaux des rapports de générations est la transmission des savoir-faire, le partage de connaissances, le développement des compétences, le processus de construction des savoirs à l'œuvre dans les divers milieux de travail. Ces divers aspects peuvent avoir un impact sur la santé des travailleurs et, selon les cas, sur les diverses clientèles qu'ils desservent, notamment dans les milieux de la santé qui nous intéressent ici. Certaines problématiques reliées à la transmission et à la formation permettent d'aborder plus ou moins directement la dimension des rapports d'âges et de générations, notamment les problématiques concernant la formation de la main-d'œuvre, le compagnonnage et le mentorat (Dolan, 1993; Houde, 1995). Notons à cet égard que la plupart des sources qui en tiennent compte sont américaines, l'enjeu démographique et intergénérationnel y ayant tenu une place plus importante qu'ailleurs dans les problématiques concernant les faits et les rapports sociaux. Dès les années 1950, par exemple, les américains étudiaient les questions du vieillissement et du cycle de vie (Chauvel, 1998, p. 90 ; Eisenstadt, 1956). En Europe, on aborde la question en regard des modifications du rapport entre activité et cycle de vie (Gaullier, 1988). On trouve aussi certaines problématiques autour du couple expert-novice, notamment celle de la dynamique des compétences (Gaudart et Weill-Fassina, 1999).

6 Des indices de l'importance des rapports entre groupes d'âge en regard des enjeux de santé et de sécurité nous sont donnés à travers les travaux sur les travailleurs vieillissants. Ces travaux font état du fait que le taux de lésions professionnelles de ces derniers est souvent moins élevé que chez les jeunes travailleurs (Laflamme et Menckel, 1995 ; Cloutier et Duguay, 1996). On s'interroge à cet égard sur l'impact positif de l'expérience acquise au fil du temps sur les attitudes prudentielles et préventives au travail ; aussi sur une utilisation plus avisée des ressources disponibles, si les conditions de travail sont favorables au développement de ces stratégies (Laflamme et Menckel, 1995 ; Salthouse, 1990 ; Warr, 1994). Une étude récente dans un Centre local de services communautaires (CLSC) de la région de Montréal fait état de divers atouts liés à l'expérience des travailleuses : elles ont acquis des savoir-faire les aidant à se prémunir contre les risques, elles mentionnent l'importance d'une fine connaissance de leurs patients, à la fois pour leur propre sécurité et pour le bien-être de ceux-ci. À ce titre, elles jugent important de transmettre ces savoir-faire à d'éventuelles remplaçantes (Cloutier et coll., 1998, p. 113).

7 Dans les pratiques de gestion en matière de vieillissement de la main-d'œuvre, on passerait d'une vision des travailleurs âgés comme une main-d'œuvre peu efficace, et qui s'exprime à travers des « stratégies d'élimination des employés vieillissants », à une autre plus positive, qui vise à intégrer les compétences spécifiques qu'offrent les travailleurs vieillissants. Ce passage s'opère notamment à la faveur d'une découverte qu'avec l'âge s'accroît « l'intelligence de la tâche ». S'il arrive que le personnel plus âgé devienne moins productif, ce serait avant tout lié à la perte de motivation (Guérin, 1991; Saba et coll., 1997 ; Gaudart et Weill-Fassina, 1999, p. 53).

Ces approches du travailleur vieillissant pourraient s'arrimer aux questions concernant les relations intergénérationnelles et interâges au travail. Quel rapport établir entre l'acquisition d'une telle intelligence de la tâche et des perspectives de transmission à des plus jeunes ? Parlant de nouveaux rôles, quels types de rôles les plus de 50 ans peuvent-ils jouer auprès des recrues? Quel est le lien entre productivité et équilibre des âges dans un 
milieu de travail ? Ce sont là des questions fort peu explorées dans les problématiques actuelles. Celles-ci sont d'ailleurs notamment tributaires d'une spécialisation des chercheurs par groupes d'âge depuis une trentaine d'années. On trouve d'un côté des spécialistes de la jeunesse, et de l'autre des experts de la vieillesse, par exemple en sociologie de la jeunesse et en gérontologie. Les liens entre cohortes ou entre générations demeurent alors abordés en périphérie des problématiques. Nous avons exploré ailleurs les soubassements culturels de ces tendances (Lefebvre, 1995, p. 129-142 ; Lefebvre, 1997).

Nous nous intéressons donc à la relation entre l'âge et l'expérience acquise, aux divers rapports aux savoirs selon l'âge et la génération, aux conditions d'échange entre les groupes d'âge et les générations au travail. Et nous abordons ici ces larges questions à travers une étude de cas, menée entre juin et octobre 1998, dans deux grands hôpitaux de la région de Montréal. Nous exposons d'abord l'hypothèse de travail, la méthodologie ainsi que les principaux résultats et nous concluons en situant les résultats en regard de perspectives actuelles.

\section{Hypothèse et méthodologie de recherche}

10 Rappelons que, dans l'ensemble du Québec, 36000 travailleurs appartenant à la fonction publique et parapublique se sont prévalus des offres de départ volontaire durant l'année 1997. Dans la région montréalaise, en décembre 1997, 5144 postes équivalents temps complet avaient été libérés de la sorte dans les seuls établissements de la santé et des services sociaux. L'objectif de ces offres spéciales de retraites prématurées était de faire face aux compressions budgétaires. Mais il s'agissait aussi de dégager une marge de manœuvre afin de favoriser la révision et la refonte de l'organisation des services, tant dans les secteurs cliniques que non cliniques (Régie régionale: 4). On voulait aussi dégager des postes pour faire place à de plus jeunes travailleurs. En 1996-1997, le statut d'occasionnel était le lot de plus de $55 \%$ des travailleurs du domaine de la santé âgés de 18 à 29 ans (Ministère de la santé et des services sociaux du Québec, 1998).

11 Le but de notre enquête préliminaire était de vérifier certaines conséquences du programme de départs volontaires de 1997 sur les milieux de travail. Les départs, plus nombreux que prévu dans tous les milieux visés au Québec, avaient-ils provoqué un certain déséquilibre des âges? Avaient-ils une incidence sur l'intégration des jeunes recrues et sur la qualité des soins? Cette situation avait-elle entraîné une perte réelle des compétences nécessaires au partage des connaissances? Pour prélever ces informations, nous avons procédé surtout par entrevues qualitatives: les résultats rendent donc compte de ce qui est perçu et ressenti par les travailleurs demeurés en place. Nous avons aussi eu accès à certaines données des services des ressources humaines pour situer ces points de vue en regard des pertes d'effectifs en 1997.

Relevons que deux enquêtes se sont intéressées à l'après-retraite des quelque 36000 employés de la fonction publique qui ont pris leur retraite durant l'année 1997-1998 dans l'ensemble du Québec. Mais elles portent sur la satisfaction et les conditions de vie des nouveaux retraités après leur départ et n'incluent aucune question sur les nouveaux rôles de formateurs que ceux-ci auraient ou non joués. La première enquête concerne l'ensemble des retraités de la fonction publique et parapublique (Dorion et coll., 1998) ; la seconde concerne en particulier les infirmières retraitées (Nadeau, 1998). 
13 Notre étude préliminaire n'est pas généralisable car elle porte sur un échantillonnage qualitatif réduit. En outre, elle a été menée dans un contexte de profonds changements organisationnels et professionnels dans les milieux de la santé. Les points de vue des travailleurs recueillis pourraient avoir changé depuis. Reste que l'intérêt de cette étude réside dans la formulation de nouvelles questions et hypothèses pour enrichir les problématiques exposées en introduction.

\section{L'échantillonnage}

L'enquête préliminaire s'est déroulée entre juin et octobre 1998, dans deux centres hospitaliers de Montréal. Le premier, que nous appellerons Établissement A, est un centre hospitalier spécialisé qui comptait, en 1997, quelque 3350 employés et cadres. L'Établissement B, centre hospitalier à vocation multiple, comptait en 1996-1997 quelque 3650 employés et cadres. Sur ces ensembles, on compte dans les deux cas environ 2800 employés (permanents à temps complet et réguliers à temps partiel), les autres étant occasionnels et sur des listes de rappel. L'offre de départ volontaire concerne les employés permanents et réguliers.

(cheurs ont effectué dix-neuf entrevues, dont quinze en milieu de travail et quatre à l'extérieur de l'établissement : dix membres du personnel infirmier (neuf infirmières et infirmiers, dont une à la retraite, et une responsable syndicale), trois représentants des directions générales des deux hôpitaux, quatre employés généraux, deux techniciennes en laboratoire. Ces employés sont âgés de 22 à 54 ans.

Nous avons procédé à des entrevues individuelles et de groupe. Ces entrevues ont été complétées par des échanges informels avec plusieurs autres personnes: une quinzaine dans chacun des hôpitaux. La plupart des entrevues n'ont pas été enregistrées, à la demande des interrogés. Le matériau comprend donc des notes prises en cours d'entrevue, mais aussi trois entrevues enregistrées d'une heure et demie, auprès d'un directeur des ressources humaines, d'une infirmière depuis longtemps retraitée et d'un jeune infirmier. On trouvera en annexe le cadre de questionnement qui a orienté les entretiens. Ceux-ci ont été complétés par des informations obtenues auprès des services aux ressources humaines, avant et après les départs volontaires dans les deux hôpitaux.

\subsection{De quelles pertes de savoir et d'expérience peut-on parler?}

17 Les médias ont fait écho de nombreuses fois du drame de la perte brutale d'expérience et de la désorganisation des milieux de la santé après les retraites de 1997. Qu'en est-il dans nos deux hôpitaux? L'examen des dossiers disponibles sur la main-d'œuvre, mais surtout les échanges avec les responsables des ressources humaines, nous permettent de développer ce qui va suivre. Faisons d'abord une remarque préliminaire : il est difficile d'analyser les enjeux démographiques de la main-d'œuvre dans nos deux institutions, car les données disponibles ne sont pas uniformes ou sont incomplètes. Aussi, la présentation que nous en faisons ici est-elle très sommaire.

Dans les hôpitaux $\mathrm{A}$ et $\mathrm{B}$, respectivement $14 \%$ et $12,5 \%$ des employés réguliers ou permanents sont partis (A : $390 ; B: 350)$. Parmi ceux-ci, on trouvait les employés les plus expérimentés, âgés de 50 ans et plus. Par ailleurs, avant les départs, les deux hôpitaux comptaient autour de $5 \%$ d'employés de moins de 29 ans ; après les départs, cette tranche 
d'âge atteignait de 13 à $15 \%$, les autres employés demeurant en poste étant dans la force de l'âge (30-50 ans).

Dans l'hôpital A, le total des départs volontaires se répartit comme suit par compétences (390 départs) : $8 \%$ cadres, 21,6 \% infirmières, $6 \%$ professionnels, $14 \%$ techniciens, $23,8 \%$ para-techniciens (comprenant aussi les employés généraux), 11,6\% bureau, $15 \%$ services auxiliaires. Dans l'hôpital B (350 départs): $5 \%$ cadres, $30 \%$ infirmières, $4 \%$ professionnels, $30 \%$ techniciens et para-techniciens, $31 \%$ services auxiliaires et bureau. Quant à la répartition par unité, elle a été établie sur la base d'entrevues avec les employés des ressources humaines, car les données documentaires ne fournissent pas ce type d'information.

\section{Conclusions préliminaires}

On ne saurait généraliser, puisque les conséquences des départs sont inégales selon les unités et les services. Les chiffres qui précèdent permettent d'énoncer une remarque générale. Il y a conjugaison de deux faits en 1997 : le départ de nombreux employés les plus âgés et l'arrivée de nombreux jeunes employés peu ou pas expérimentés. Peut-on, à partir de notre enquête exploratoire, en conclure à l'évidement des milieux, au démantèlement des équipes de travail et aux brisures des lignes de transmission des savoirs? Il semble qu'il faille distinguer selon les caractéristiques des unités, les spécialisations, les quarts de travail et les types de savoir en jeu.

1. Certaines unités se sont vidées. Surtout celles où se trouvait une concentration d'employés parvenus à l'âge de la retraite, donc plus âgés. Il s'agissait surtout des services prisés par ces travailleurs plus anciens : par exemple la gynécologie, les prématurés, la clinique externe, le bloc opératoire, etc. Ces unités ont été démantelées. D'autres unités se sont retrouvées sans employés d'expérience, dans le cas où les équipes y étaient entrées par vagues; elles étaient donc composées de membres de la même ancienneté, par exemple les salles d'opération, dans les deux établissements ;

2. Dans d'autres cas, trois ou quatre employés sur huit ou neuf membres sont partis ; l'équipe se trouva donc cassée en deux ;

3. Selon les quarts de travail, on trouve une plus grande concentration de recrues, surtout de nuit ; les employés d'expérience ne s'y trouvent donc pas en nombre suffisant pour assurer un transfert de connaissances ;

4. On parle aussi de départs individuels irremplaçables. Dans l'hôpital A, centre hospitalier spécialisé, certaines spécialisations étaient maîtrisées par un seul individu ; dans l'hôpital B, une interrogée fait explicitement allusion au poste d'un employé perfusioniste dont on a retardé le départ par une «mesure spéciale » négociée avec le ministère de la Santé, afin qu'il puisse entraîner la nouvelle recrue ;

5. Parmi les préretraités, certains n'ont consigné nulle part des orientations, des plans de travail et autres procédés dans le milieu, structurés avec le temps. Il peut s'agir de l'emplacement d'une information ou d'un dossier, d'une stratégie d'action, etc. Cette mémoire déclarative individuelle, selon les termes de la psychologie cognitive, i.e. pouvant être explicitée mais appartenant à un individu, est forcément susceptible d'être perdue advenant le départ de cet individu. Une interrogée, technicienne, raconte les conséquences du départ d'une réceptionniste de son département:

«C'est fou, elle n'a même pas eu le temps d'entraîner la remplaçante. Celle-ci ne trouvait pas l'emplacement des dossiers, des informations précises utiles pour diriger judicieusement les appels ou les visiteurs ; tout était à refaire ». 
Les contenus de cette mémoire déclarative individuelle s'en sont donc allés, et dans beaucoup de cas, aucun de ces contenus ne pouvait être transmis autrement qu'à travers une relation avec les nouveaux travailleurs (Girod, 1995 ; Chapelle, 1999).

2 Quant au diagnostic global de perte de mémoire, diffusé ici et là dans les médias depuis 1997, il n'est pas tout à fait exact. En effet, il est resté dans le milieu des travailleurs comptant plus de vingt ans d'expérience. Dans le contexte qui a suivi, cependant, ceux-ci n'avaient pas le temps d'accueillir les nouveaux. Ils faisaient généralement face à des contraintes d'efficacité qui faisaient en sorte qu'elles et ils n'avaient pas le temps de s'occuper des nouveaux, des plus jeunes. Rappelons que le nombre de recrues, après les départs volontaires, a plus que triplé dans les deux hôpitaux étudiés, se situant entre 13 et $15 \%$. Ajoutons que comme les plus jeunes recrues sont précaires, ou proviennent de listes d'appel, la formation est souvent à refaire puisqu'ils présentent une mobilité très grande.

23 En outre, comme l'un des objectifs de l'offre massive des départs volontaires était la réorganisation du travail, celle-ci n'a pas tardé. Si bien qu'il semble que même au sein du personnel expérimenté demeuré en poste, il y a eu une certaine déroute. Sans compter le mouvement de personnel important auquel ont donné lieu les affichages de poste. Comme plusieurs emplois très recherchés et occupés par les plus anciens avaient été libérés, nombreux sont les employés demeurés en emploi qui y aspiraient à leur tour.

24 Les employés dans la force de l'âge affrontaient donc au moins trois difficultés : faire face à de nouvelles conditions organisationnelles ou à un nouveau poste, s'adapter et accueillir un nouveau personnel et vivre le départ de nombreux collègues. Une étude effectuée dans la région de Québec concluait à un haut degré de détresse psychologique chez les infirmières demeurées à l'emploi des établissements au lendemain des départs de 1997 (Bourbonnais et coll., 1998). Dans les deux établissements, les interrogés travaillant aux ressources humaines faisaient ce constat commun :

«Il est vrai que pour opérer un changement profond des manières de faire, il faut procéder rapidement, afin de surmonter les résistances et l'inertie propres à un milieu. Mais nous aurions dû, à travers cette stratégie de changement, mieux évaluer les facteurs de transition, parmi lesquels des besoins de formation du nouveau personnel. À cet égard, nous aurions pu prendre un peu plus de temps pour mener l'ensemble de l'opération ».

Quant aux recrues ou aux nouveaux, certains arrivent dans des unités quasiment vidées, travaillent sur des quart de travail où il $\mathrm{y}$ a peu de perspectives d'intégration et d'initiation. Les conséquences de cette situation sur la santé et la sécurité du personnel n'ont pu être mesurées avec précision dans le cadre d'une enquête couvrant une durée limitée ; toutefois, dans les entrevues, on notait de nombreuses mentions des facteurs suivants : insécurité, stress, haut taux d'absentéisme, démotivation (jusqu'à envisager de quitter la profession ou le milieu). Il serait à ce titre pertinent d'analyser les dossiers des hôpitaux de manière comparative, de 1996 à aujourd'hui, afin de préciser les conséquences exactes qu'ont provoquées tous ces changements de 1997 sur les employés, à court et à moyen terme. Mentionnons que cinq employés interrogés ont émis l'opinion selon laquelle l'évidement radical d'employés d'expérience dans certains milieux mettait la santé des patients en danger (bloc opératoire ou banque de sang par exemple).

Rappelons à ce sujet des constats assez classiques dans le champ de la démographie organisationnelle. Si une main-d'œuvre massivement vieillissante peut effectivement faire obstacle aux innovations, son remplacement brutal et rapide comporte des effets de 
déstabilisation tout aussi négatifs, en provoquant par exemple beaucoup d'anxiété au sein du personnel. Comme le rapportent Séguin et Pommainville,

« une distribution relativement linéaire et uniforme de la permanence de la maind'œuvre semble procurer le meilleur équilibre entre l'innovation, l'adaptation et la stabilité dans les organisations. Cela implique que les gestionnaires de nos organisations auraient avantage à établir un système de planification des ressources humaines qui permette de scruter chaque mouvement migratoire de leur personnel afin de pouvoir assurer un certain équilibre démographique dans leur entreprise » (Séguin et Pommainville, 1988, p. 34).

27 Les auteurs observent aussi que les milieux syndiqués présentent à cet égard des défis particuliers, puisque les protections qu'ils négocient sont proportionnelles au degré d'ancienneté des employés, et de ce fait ne favorisent pas d'abord l'équilibre démographique des milieux où ils sont implantés.

\section{Experts, novices et défis d'apprentissage en soins infirmiers}

Quant à la valeur de l'expérience acquise et aux relations entre groupes d'âge de travailleurs, seule la dizaine d'entrevues des infirmières et infirmiers nous procure un matériau suffisant pour étayer davantage nos résultats. Un jeune infirmier de 23 ans, qui travaille dans l'hôpital A, tient ces propos révélateurs :

«La formation est garochée, c'est un québécisme, mais c'est comme ça. Y’en a une vingtaine qui ont été engagés, ils ont eu une semaine de formation, c'est tout ; après ça, ils sont lancés dans le milieu de travail ; alors le risque d'erreur est plus grand à ce moment-là. Tandis que quand on avait tous les gens qui sont retraités, c'est des gens avec de l'expérience qui ont des acquis, qui ont, à ce moment-là, y'ont pas à apprendre tout ce qu'un jeune aurait à apprendre. Puis pour former un infirmier, moi ça fait deux ans que je travaille puis je suis encore bien insécure... Ça fait un an et demi là mais ça fait deux ans avec deux hôpitaux, pis je suis encore bien insécure dans ma profession t'sé; j'y vais à pas de tortue là, j'veux apprendre mais apprendre à mon rythme pis c'est comme ça j'pense pour la plupart ; ça prend deux ou trois ans pour préparer un bon infirmier, pis encore là ça varie d'un milieu de travail à l'autre [...]. Quand ça fait cinq à sept ans, tu es encore considéré comme un jeune infirmier, en tout cas tu te fais surveiller disons quand tu fais des soins pis tout ça ; les plus vieux te regardent, te regardent aller, ils t'encadrent plus. »

Ces propos évoquent la valeur de l'expérience acquise dans les soins infirmiers, de même que les difficultés rencontrées dans le contexte des départs. Une infirmière de 49 ans va dans le même sens :

«Les travailleurs en place depuis de nombreuses années ont une connaissance terrain. Ils maîtrisent les techniques de soins et ont été confrontés à une vaste gamme de situations. C'est donc à eux que devrait revenir le rôle de servir de guides et de conseillers auprès de jeunes qui entrent dans les milieux hospitaliers. Malheureusement, dans le contexte actuel, les travailleurs d'expérience sont largement absents ou pas assez nombreux. »

Trois jeunes infirmières embauchées suite aux départs massifs nous ont dit ne plus savoir vers qui se tourner lorsqu'elles ont des questions à poser. L'une d'entre elles précise :

«Dans notre hôpital, les quelques infirmières d'expérience qui restent sont dépassées par la situation et n'ont pas de temps à nous consacrer. Dans plusieurs unités, lorsqu'elles nous voient venir, elles se disent: «Oh non! Pas encore des nouvelles!». Dans le fond, on les comprend, mais il y a des choses qu'on a besoin de 
vérifier avec des professionnelles d'expérience. Parfois, c'est la façon de faire fonctionner l'équipement, ou des techniques propres à une condition particulière... Mais souvent, on a peur de déranger, donc on se débrouille entre nous. À plusieurs, on arrive habituellement à trouver des solutions. "

31 Deux des jeunes infirmières bachelières nouvellement embauchées par l'un des établissements ont raconté s'être retrouvées seules à diriger un important département de l'hôpital, alors qu'elles cumulaient seulement quatre mois d'expérience. Une autre jeune infirmière de 26 ans nous a confié avoir eu, après un mois de travail seulement, à superviser des préposé(e)s aux bénéficiaires et infirmières auxiliaires qui comptaient dixhuit à vingt ans d'expérience :

«C'est absurde, on nous propulse dans des postes de responsabilité sans s'être assuré que nous ayons la capacité de remplir la commande ». 1993).

\section{Savoirs et dynamiques de transmission}

Il ressort de nos entrevues que l'expérience acquise est très importante pour la profession infirmière, plus spécialement pour les soins directs aux patients. Quant à la nature des savoirs qui nécessitent des stratégies de transmission, des observations participantes 
seraient nécessaires afin de mieux préciser. Les entrevues nous indiquent cependant des défis spécifiques.

Les jeunes recrues font face au défi d'adaptation aux lieux physiques: trouver l'emplacement des dossiers, des outils, des informations. Ils doivent aussi «observer » certaines techniques de soins avant de les appliquer adéquatement. Ils doivent donc fréquemment demander où se trouvent les objets en question et regarder des employés plus expérimentés donner des soins :

" Après un an, tu deviens habitué avec les lieux physiques et puis tout ça... puis les techniques tu en as vues, ton expérience se crée tranquillement... alors eux autres (les travailleurs d'expérience), ils voient arriver les nouveaux, c'est comme une surcharge de travail. »

Il y a aussi des défis d'organisation d'un travail où s'enchaînent plusieurs tâches précises :

«Ils sont obligés de te surveiller, de dire, t'sé tout le temps, par exemple, as-tu fait ton prélèvement? Ils te le rappellent et ils te surveillent; as-tu relevé tes ordonnances? Tu dis oui mais ils doivent checker quand même; il faut noter le nombre de liquide ingéré par un patient; tu sais, c'est banal, mais une nouvelle avait oublié, tu sais, et l'employé d'expérience l'a traitée d'imbécile... ! ».

Une organisation de la tâche qui comporte aussi des dimensions temporelles, par exemple dans la préparation d'un traitement: administrer un médicament, préparer le patient, prévoir le temps de ces soins préparatoires, etc.

Les soins infirmiers sont en outre des savoirs complexes et qui nécessitent par exemple une approche analytique et évaluative des divers aspects :

«Moi, raconte une infirmière expérimentée, j'ai toujours dit que le plus grand danger, c'est de ne pas savoir qu'on ne sait pas, surtout dans le domaine de la santé. Tu peux par exemple donner un médicament, c'est prescrit de donner telle dose, à telle fréquence, etc. Tu le donnes, mais si tu ne connais pas les effets secondaires de ce médicament, ou si tu connais pas les interférences de ce médicament avec un autre médicament, tu peux avoir des problèmes. »

La dimension humaine et relationnelle semble s'approfondir au fil de l'expérience :

« Des aspects importants de l'expérience des anciennes, dans l'expérience précieuse qu'elles accumulaient au cours des ans, c'était leur capacité d'empathie, la capacité de comprendre les gens puis d'en venir à prévoir les réactions, les réactions des patients, les réactions de la famille. Elles avaient accumulé tout un capital humain qui, à mon avis, était d'une grande richesse et qu'on ne peut acquérir qu'avec les années » (infirmière à la retraite).

43 Selon les qualités personnelles des travailleurs et les besoins de la tâche, des activités informelles de transmission peuvent se mettre en place :

«Souvent les plus vieux, je ne parle pas de tout le monde, il y en a qui sont extraordinaires, mais la grosse majorité, quand ils voient arriver un nouveau sur l'étage, ils disent: Ah il faut le checker, j'ai pas le temps pour ça !... S'ils voient qu'il pose beaucoup de questions, ils chialent... Ils ont pas le temps de s'attarder à montrer, à surveiller quelqu'un, mais ils savent qu'ils doivent le faire... C'est leur rôle ben c'est pas écrit, tu sais, mais ils savent qu'ils vont avoir des problèmes s'ils ne surveillent pas. Comme dans le cas de soins à donner à un polytraumatisé, par exemple...»

$44 \mathrm{Au}$ plan de la dynamique générale de transmission et d'échange de savoirs, la comparaison entre nos résultats et ceux d'enquêtes menées subséquemment permettent d'attirer l'attention sur une triple dynamique d'apprentissage (Lefebvre et coll., 1999): 
l'apprentissage entre pairs s'opère à travers des stratégies d'urgence et de débrouillardise; l'accompagnement par un employé expérimenté est nécessaire, et l'organisation devrait laisser une marge de manœuvre pour qu'il soit possible; l'accompagnement plus large et volontaire par des employés au seuil de la retraite pourrait renvoyer à des qualités précieuses pour les plus jeunes (patience, rapport moins compétitif, expérience relationnelle, etc.).

Une autre étude dans des milieux de travail comportant des exigences de maîtrise technologique élevées montre par ailleurs que les formateurs les plus indiqués pour les plus jeunes étaient non pas au seuil de la retraite mais d'âge moyen. Ces formateurs, en effet, devaient être à la fois expérimentés et proches de l'univers référentiel, scolaire et culturel des plus jeunes pour arriver à leur communiquer adéquatement leur expérience (Lefebvre et coll., 1999).

\section{Conclusion}

Cette étude de cas dans deux établissements hospitaliers de la région de Montréal permet donc de préciser les hypothèses selon lesquelles les départs massifs à la retraite auraient eu des impacts négatifs sur les milieux. Retenons que ceux-ci varient selon les unités, les services et les quarts de travail : dans certaines équipes de travail, on observe un plus grand déséquilibre entre les générations de travailleurs. Les entrevues qualitatives indiquent la valeur de l'expérience acquise, surtout en matière des soins infirmiers directs aux patients. Les interrogés reconnaissent en général que les stratégies organisationnelles n'ont guère laissé place à des enjeux de transmission aux nouvelles recrues, que ceux-ci dépendent surtout de l'initiative, de la disponibilité et de la générosité des travailleurs en place. La combinaison, dans un même temps, de nombreux départs de travailleurs âgés et de l'arrivée de plusieurs recrues n'a pas favorisé les échanges. En termes de santé et de sécurité, les interrogés ont évoqué les facteurs de stress et d'anxiété chez les employés, et les risques encourus par les patients exposés à des employés inexpérimentés et sans surveillance adéquate. Situons maintenant ces aspects dans une perspective plus large.

Une valorisation de l'expérience et de sa transmission doit nécessairement s'intégrer à l'intérieur du cadre plus large d'une gestion de la mémoire d'une entreprise ou d'une institution. On référera à ce sujet aux travaux qui font usage des sciences cognitives pour différencier les types et les sources diverses de savoir dans un milieu. Ces travaux mentionnent à la fois l'importance de la consignation des informations et procédures mémoire déclarative - et celle des savoirs tacites, non dits, informels - mémoire procédurale. La transmission de ces mémoires du travail est précaire, dépendant en partie d'une consignation par écrit, en grande partie sur les relations et les affinités entre travailleurs (Chapelle, 1999, p.53). Or, les contextes actuels de travail rendent ces échanges difficiles, tel que nous venons de l'illustrer. Nous estimons par conséquent que la prise en compte de cet enjeu doit devenir l'une des priorités de la gestion des institutions publiques et para-publiques : il faut envisager des moyens de conserver les connaissances de ceux et celles qui partent. Peut-on par exemple trouver un compromis entre les stratégies d'ébranlement du milieu pour mieux effectuer des changements, et une attention à la continuité ? Du côté syndical, est-il possible de viser un compromis, entre le principe d'égalité - qui impose, le cas échéant, des offres de retraite non sélectives - et l'attention au principe de continuité dans l'institution? C'est là un 
impératif que tend à illustrer notre étude de cas. Toutefois, il est certain que cette continuité ne peut être assurée sans prêter attention à l'importance des interactions entre travailleurs d'expériences différentes, ce qui est un processus graduel et coûteux. En outre, la reconnaissance de cette nécessité d'assurer une transmission de l'expérience repose sur le fait que tous les types de savoir ne sont pas changeants ou révisables au même titre. Des savoir-faire pratiques, par exemple, ont une certaine pérennité que n'ont pas les savoirs plus théoriques (Bernadou, 1999).

À cet égard, en mars 1999, la FIIQ a présenté au ministère de la Santé et des Services sociaux un rapport ad hoc du Groupe de travail sur la planification stratégique de la main-d'œuvre infirmière où les dimensions de la formation en milieu de travail et du tutorat tiennent une place centrale. On évoque aussi le drame de l'effritement du sentiment d'appartenance, du fait de l'éclatement des équipes de travail (FIIQ, 1999). Une problématique émergente dans le champ d'étude des travailleurs vieillissants a trait notamment à la satisfaction qu'ils retireraient d'exercer de nouveaux rôles de formateurs (Saba, 1995). On peut mettre cette possibilité en lien avec la Loi 106 du gouvernement du Québec, concernant la retraite progressive et la retraite anticipée, mais qui doit être nommément intégrée dans une convention collective pour être envisageable pour un employé. Le projet de loi fait une allusion explicite à la possibilité de transmettre des connaissances aux recrues, à travers une stratégie de retraite progressive, et l'avantage que pourraient y trouver les institutions.

Enfin, tout en jugeant utile de mieux documenter les liens entre apprentissage, dynamique de compétence et âge/génération, il semble probable que cette différenciation doive s'inscrire dans une dynamique collective de construction des savoirs :

«Group mentoring will come into vogue as the traditional one-on-one relationship gives way to groups of professional mentoring one another.» (Dolan, 1993; Chatigny, 2000).

\section{BIBLIOGRAPHIE}

Attias-Donfut, C. (1991). Générations et âges de la vie. Que sais-je ? 2570, PUF, Paris, 126 p.

Bernadou, A. (1999). Le savoir en action. L'exemple médical. Sciences Humaines, Hors Série : La dynamique des savoirs, 24, 26-28.

Bourbonnais, R., Brisson, C., Comeau, M., Laliberté, D., Malenfant, R., Vézina, M., Viens, C. (1998). La transformation du réseau de la santé: mesure des caractéristiques du travail et de la santé des infirmières de l'agglomération de Québec. Équipe de recherche RIPOST, Université Laval et Direction de la Santé publique de Québec, 55 p.

Chapelle, G. (1999). La mémoire de l'entreprise. Sciences Humaines, Hors Série : La dynamique des savoirs, 24, 52-53.

Chatigny, C. (2000). La construction et l'usage des ressources dans le processus d'apprentissage en situation de travail : analyse comparative de deux métiers. Thèse de doctorat d'ergonomie. Conservatoire national des Arts et Métiers (en rédaction). 
Chauvel, L. (1998). Le destin des générations. Structure sociale et cohortes en France au XXe siècle. PUF, Paris, $301 \mathrm{p}$.

Cloutier, E., Duguay P. (1996). Impact de l'avance en âge sur les scénarios d'accidents et les indicateurs de lésions dans les secteurs de la santé et des services sociaux, de l'administration provinciale et de l'administration municipale. IRSST, Montréal, RR-119.

Cloutier, E., David, H., Prévost, J., Teiger, C. (1998). Santé, sécurité et organisation du travail dans les emplois de soins à domicile. IRSST, Montréal, R-202, 148p.

Dejours, C. (1993). Travail, usure mentale, Centurion/Bayard édition, Paris.

Dolan, T.C. (1993). Mentoring in the 1990's. Healthcare Executive, 8, 6.

Dorion, M., Fleury, C., Leclerc, D.P. (1998). Que deviennent les nouveaux retraités de l'État ? Rapport final. Rapport rédigé dans le cadre du cours SOC-18879, Laboratoire sociologique sous la supervision de Simon Langlois, Jean-Jacques Simard et Jean-François Simard, Département de sociologie, Université Laval, 245 p.

Eisenstadt, S. (1956). From Generation to Generation : Age Groups and Social Structure. Glencoe, Free Press.

FIIQ (1999). Orientations de la FIIQ relativement à la planification stratégique de la main-d'œuvre infirmière. Conseil fédéral, D6, $14 \mathrm{p}$.

Gaudart, C., Weill-Fassina, A. (1999). L'évolution des compétences au cours de la vie professionnelle : une approche ergonomique. Formation Emploi, numéro spécial Activités de travail et dynamique des compétences, 67, 47-62.

Gaullier, X. (1988). La deuxième carrière : âges, emplois, retraites. Éditions du Seuil, Paris, 408 p.

Girod, M. (1995). La mémoire organisationnelle. Revue française de gestion, 105.

Guérin, G. (1991). Les pratiques de gestion en matière de vieillissement. Dans Vieillir en emploi, eds R. Blouin, p. 51-89. Les presses de l’Université Laval, Sainte-Foy.

Houde, R. (1995). Des mentors pour la relève. Méridiens, Montréal.

Huuhtanen, P., K. Ahola, Leino, T. (1997). Improving Cooperation At Work Betwwen Generations. In From Experience to Innovation, IEA 97 Proceedings of the $13^{\text {th }}$ Triennal Process of the International Ergonomics Associations, Tampere, Finlande, 5, p. 442-444.

Laflamme, L., Menckel, E. (1995). Aging and occupational accidents. A review of the literature of the last three decades. Safety Science, 21, 145-161.

Lefebvre, S. (1995). Générations contemporaines, itinéraires et solidarités. Dans J., Grand'Maison, L., Baroni et J.-M. Gauthier (dir.) Le défi des générations. Enjeux sociaux et religieux du Québec d'aujourd'hui, Montréal, Fides, p. 97-181.

Lefebvre, S. (1997). Rapports de générations. Une conjoncture socio-économique et culturelle. Cahiers internationaux de sociologie, CII, 183-198.

Lefebvre, S., Cloutier, E., Chatigny, C. (1999). La transmission des savoirs professionnels dans deux milieux de travail participant au régime de qualification géré par Emploi-Québec. Groupe de recherche sur la transmission en milieu de travail, Faculté de théologie, Université de Montréal, Rapport remis à Emploi-Québec dans le cadre d'une étude sur des expériences de compagnonnage dans les milieux de l'usinage et des cuisines d'établissement.

Lichtenberger, Y. (1999), Compétence, organisation du travail et confrontation sociale, Formation Emploi, 67, 93-107. 
Ministère de la santé et des services sociaux du Québec (1998). Répartition des effectifs syndiqués selon le statut occupationnel et l'âge : année 1996-1997; et Programmes de départ volontaire à la retraite (rapport selon les statistiques reçues en date du 20 août 1998).

Nadeau, I. (1998). Profil psycho-socio-économique de l'infirmière retraitée. Rapport de recherche. Regroupement des Infirmières et Infirmiers retraités (RIIR) et Fédération des infirmières et infirmiers du Québec (FIIQ), 48 p.

Régie régionale de la santé et des services sociaux Montréal-Centre (1997). Le défi de l'accès, l'organisation des services de santé et des services sociaux sur l'île de Montréal 1998-2001. 192 p.

Ritchie, N., Connolly, M. (1993). Mentoring in public sector management : Confronting accountability and control. Management Education \& Development, 24, 3, 266-279.

Saba, T. (1995). Les aspirations insatisfaites des professionnels syndiqués de 50 ans ou plus : nature et gestion. PhD, Université de Montréal.

Saba, T., Guérin, G. Wils, T. (1997). Gérer l'étape de fin de carrière. Gestion 2000, 165-181.

Séguin, F., Pommainville, R. (1988). La démographie organisationnelle : l'émergence de nouveaux défis. Gestion, 30-35.

\section{ANNEXES}

\section{Annexe 1 Cadre d'entrevues}

Thèmes généraux sur les âges et les générations à aborder dans les entretiens formels et informels

Bloc 1. Parlez-nous de votre travail, après les départs volontaires dans votre milieu?

Bloc 2. Quelle est votre vision des divers groupes d'âge de travailleurs (20-35 ans ; 35-45 ans ; 45-55 ans ; 55 ans et plus) ; et parlez-nous des liens entre eux ?

Bloc 3. Comment sont vus les travailleurs plus jeunes dans le milieu?

Quelles sont leurs forces et leurs faiblesses, si on les compare aux plus âgés ?

Comment sont les stratégies d'insertion des jeunes?

Comment ces derniers sont-ils accueillis?

Quels types de relations privilégiées nouent les jeunes dans le milieu?

Bloc 4. Comment sont vus les travailleurs plus âgés dans le milieu?

Quelles sont leurs forces et leurs faiblesses, si on les compare aux jeunes?

Jouent-ils des rôles spécifiques selon leur âge, de nouveaux rôles?

Lesquels?

\section{RÉSUMÉS}

Dans les recherches sur la santé et la sécurité au travail, les rapports entre générations de travailleurs demandent à être étudiés davantage. L'un des enjeux est la transmission des savoirfaire pouvant avoir un impact sur la santé des travailleurs et, selon les cas, sur leurs clientèles. Ces questions sont abordées à travers une étude de cas menée en 1998 dans deux hôpitaux de la région de Montréal, après les mises à la retraite de 1997 et l'arrivée de nombreuses recrues. Les impacts négatifs varient selon les unités et les quarts de travail; on reconnaît la valeur de 
l'expérience acquise pour les savoirs infirmiers, qui doit être combinée aux savoirs des recrues ; on reconnaît également que les stratégies organisationnelles n'ont guère laissé de place à ces échanges, renvoyés aux initiatives informelles des travailleurs. En termes de santé et de sécurité, on note les facteurs de stress et d'anxiété chez les employés, et les risques encourus par les patients livrés à des employés inexpérimentés et sans surveillance adéquate.

As regards research on health and security in the workplace, the relationships among various generations of workers require further study. One crucial point is the transmission of knowledge and skills that may affect the health of workers or, possibly, those in their care. These subjects are broached in a case study pursued in 1998 in two hospitals of the Montreal region following the wave of early retirement cases in 1997 and the arrival of numerous newly hired employees. The negative impact has been found to vary according to the unit and work quarter studied. The value of experience with regards to nursing skills has been recognized; it is clear that the theoretical knowledge of the newly hired employees must be combined with experience. It has also been shown that organizational strategies have left little room for any exchange, which is left to the informal initiatives of the workers themselves. As regards health and security, it has been found that anxiety and stress among employees are an important factor, and that the risks run by patients left in the care of inexperienced employees and without adequate surveillance remain a concern.

En las investigaciones de salud y seguridad en el trabajo, se debe estudiar más las relaciones entre generaciones de trabajadores. Uno de los desafíos es la transmisión de las pericias que pueden tener un impacto en la salud de los trabajadores y, en ciertos casos, sobre su clientela. Se aborda estas cuestiones en un estudio llevado en el año 1998 en dos hospitales de la región de Montreal, después de jubilaciones en el año1997 y la llegada de muchos empleados nuevos. Los impactos negativos varian según las unidades y los turnos de trabajo; se reconoce el valor de la experiencia para los saberes enfermeros, que debe combinarse con los saberes de los empleados nuevos ; también se reconoce que las estrategias organizacionales no han dejado lugar para estos intercambios, devueltos a las iniciativas informales de los trabajadores. En términos de salud y de seguridad, se nota los factores de estrés y de anxiedad con los empleados, y los riesgos incurridos por los patientes abandonados a empleados sin experiencia y sin supervisión adecuada.

\section{INDEX}

Mots-clés : retraite, milieu hospitalier, générations, transmission, savoirs

Palabras claves : jubilación, medio hospitalario, generaciones, transmisión, saberes

Keywords : retirement, hospital, generations, transmission, knowledges

\section{AUTEUR}

\section{SOLANGE LEFEBVRE}

GRPSR Transmission en milieu de travail, Faculté de théologie, Université de Montréal, Lefebsol@magellan.unmontreal.ca 